\title{
Lost and found: cardiac stem cell therapy revisited
}

\author{
Kenneth R. Chien \\ Massachusetts General Hospital Cardiovascular Research Center, Department of Cell Biology, Harvard Medical School, \\ Harvard Stem Cell Institute, Boston, Massachusetts, USA.
}

\begin{abstract}
Several clinical trials of bone marrow stem cell therapy for myocardial infarction are ongoing, but the mechanistic basis for any potential therapeutic effect is currently unclear. A growing body of evidence suggests that the potential improvement in cardiac function is largely independent of cardiac muscle regeneration. A study by Fazel et al. in this issue of the JCI provides evidence that bone marrow-derived c-kit ${ }^{+}$cells can lead to an improvement in cardiac function in mutant hypomorphic c-kit mice that is independent of transdifferentiation into either cardiac muscle or endothelial cells, but rather is associated with the release of angiogenic cytokines and associated neovascularization in the infarct border zone (see the related article beginning on page 1865). These findings suggest the potential therapeutic effect of specific paracrine pathways for angiogenesis in improving cardiac function in the injured heart.
\end{abstract}

The physician without physiology flounders along in an aimless fashion, never able to gain an accurate conception of disease, practicing a sort of popgun pharmacy, bitting now the malady and again the patient, he bimself not knowing which.

- William Osler

Over the past few years, several hundred patients have been treated with various forms of bone marrow stem cell therapy during acute myocardial infarction, ranging from direct intracoronary injection of unfractionated bone marrow-derived cells to treatment with agents that mobilize circulating hematopoietic stem cells (refs. 1-5; for review, see refs. 6,7$)$. Based on dramatic, early studies in experimental animals $(8,9)$, these cell-based therapy clinical trials were quickly initiated, with the hope that augmenting the number of injected or circulating hematopoietic stem cells would lead to the regeneration of the myocardium that had been lost during the acute ischemic event. The large, unmet clinical need and the promise of stem cell therapy formed the basis for this rapid clinical translation. Despite great interest in cell-based therapy for heart failure, the suitability of this approach remains as intriguing as ever. Although initial anecdotal, uncontrolled clinical reports suggested that there might be a therapeutic benefit, there now appears

Conflict of interest: The author has declared that no conflict of interest exists.

Citation for this article: J. Clin. Invest. 116:1838-1840 (2006). doi:10.1172/JCI29050. to be a growing consensus that the mechanism for any potential benefit is unknown and there is little evidence to support the onset of cardiac muscle regeneration following any type of clinical bone marrow cell therapy (for review, see ref. 7). Experimental studies now suggest that the transferred cells are largely eliminated from the heart within the first 24-48 hours after intracoronary infusion (10). Compounding the difficulty, the results of 4 separate double-blind, randomized, placebo-controlled trials have been ambiguous, either showing a modest effect (the REPAIR-AMI [Reinfusion of Enriched Progenitor Cells and Infarct Remodeling in Acute Myocardial Infarction] trial reported a 3\% improvement in ejection fraction versus controls; for review see ref. 6), short-lived benefit (results of the BOOST II [Bone Marrow Transfer to Enhance ST-Elevation Infarct Regeneration] trial showed no significant difference between control and treated individuals at 18 months; ref. 2), or no improvement in global cardiac function (STEMI [ST-Elevation Acute Myocardial Infarction; ref. 3]; and ASTAMI [Autologous Stem Cell Transplantation in Acute Myocardial Infarction; ref. 4] trials; for review see ref. 6). Although previous studies have reported that the administration of hematopoietic growth factors can mobilize hematopoietic stem cells and thereby augment cardiac function by regenerating myocardium following myocardial infarction (8), the results of the large scale clinical Regenerate Vital Myocardium by Vigorous Activation of Bone Marrow Stem Cells (REVIVAL-2) trial designed to test this concept have also been negative
(5). Independent attempts from many labs to reproduce the results of the initial animal studies (9) that claimed a robust effect of bone marrow stem cell therapy in acute myocardial infarction with respect to cardiac muscle regeneration have also been negative (11-13). Although there has been continued evidence to suggest an improvement in cardiac contractile function in the short term following multiple forms of cell therapy in the post-myocardial infarct setting, the basis for this effect remains unclear (14). Given the lack of an unequivocal proof of concept in either the clinical or experimental arena, the field of cardiovascular stem cell therapy could be viewed as lost in translation (15). Unfortunately, without a proven physiological underpinning for any potential therapeutic signal, it has become difficult to optimally redesign the clinical studies. Conceptually, a number of known variables could account for the potential therapeutic effects of cell therapy on cardiac function following myocardial infarction, both cardiomyocyte cell autonomous (cell survival/death pathways, hypertrophy, calcium cycling, cytoskeletal, etc.) and non-cell autonomous (neoangiogenesis, scar formation, chamber dilation, hemodynamic workload, etc.). As noted by Osler over a century ago, the transition from "popgun pharmacy" to targeted therapy first requires a firm understanding of the pathophysiological basis of the desired therapeutic endpoint.

\section{Paracrine angiogenic pathways for cardiac repair by bone marrow precursors}

In the current issue of the JCI, Fazel and coworkers report on their use of genetically engineered mouse models toward this goal (16) (for brief diagrammatic overview, see Figure 1). Utilizing hypomorphic c-kit mutant mice (compound heterozygote $\mathrm{Kit}^{\mathrm{W}} / \mathrm{Kit}^{\mathrm{W}-\nu}$ mice) that harbor an intrinsic defect in hematopoietic stem cell mobilization, the authors noted an accelerated progression to dilated cardiomyopathy following acute myocardial infarction versus control mice, which is associated with a decrease in neoangiogenesis in the injured myocardium. By reconstituting the bone marrow of irradiat- 
A

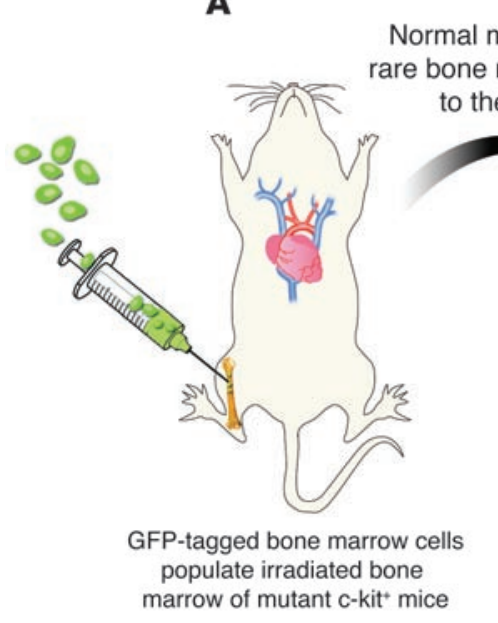

B

\section{(}

C

C

\section{Coronary ligation and \\ myocardial infarction}

(n)

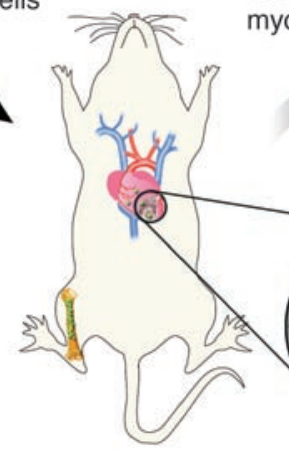

Bone marrow-derived GFP-tagged c-kit' cells become resident in the heart
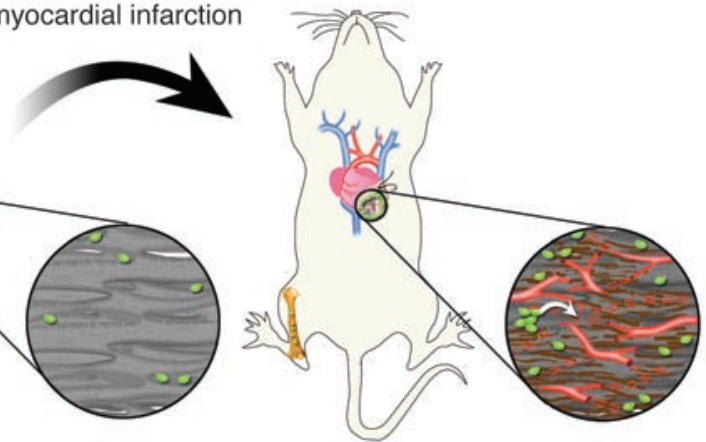

Marked migration of bone marrowderived cells to the infarct border zone leads to secretion of angiogenic cytokines and neovascularization

\section{Figure 1}

Bone marrow transplantation of mutant $c$-kit mice partially rescues dilated cardiomyopathy following acute myocardial infarction via neovascularization (16). (A) Compound heterozygote mutant $K i t^{W / K i t}{ }^{W-v}$ mice are irradiated and the bone marrow reconstituted with GFP-tagged bone marrow precursors derived from wild-type mice. (B) A small number of the wild-type c-kit ${ }^{+}$GFP-tagged bone marrow-derived cells migrate into the heart of the mutant $c-k_{i t}+$ mice. (C) Coronary ligation results in an acute myocardial infarction in the mutant mice, with the marked migration of the wild-type GFP-tagged c-kit ${ }^{+}$cells into the infarct border zone. Compared with mutant $c-k i t$ mice that have not had reconstitution with wildtype bone marrow precursors, there is a markedly smaller extent of chamber dilation and associated dysfunction in the wild-type transplanted mutant $c$-kit mice. The beneficial effect is not associated with transdifferentiation into cardiac myocytes or vascular cells but rather is associated with the release of angiogenic cytokines and an increase in neovascularization.

ed wild-type mice with GFP-tagged cells, the authors determined that there are only rare c-kit ${ }^{+}$cells in the heart at baseline and the vast majority of the cells that are resident in the heart following acute myocardial infarction are bone marrow derived, suggesting that it might be possible to rescue the cardiac defect in the mutant mice via bone marrow reconstitution with wild-type bone marrow cells. The authors go on to document this rescue of the heart phenotype with wild-type bone marrow cell transplantation, which is associated with an induction of angiogenic cytokines and enhanced neoangiogenesis. Taken together, these results suggest that bone marrow-derived progenitors can have a marked influence on cardiac repair by promoting neoangiogenesis via paracrine signaling pathways as opposed to directly transdifferentiating into either cardiac muscle or vascular cells. The studies identify clearly, for the first time, a defined paracrine role for cell-based therapy in the injured heart following myocardial infarction.

\section{Experimental and clinical implications}

On the experimental front, the study (16) raises several critical issues. First and foremost is whether these studies in a mutant mouse model system have any direct bearing on ongoing human clinical trials. It will become critical to rigorously quantitate changes in specific paracrine factors following cell therapy in small and large animal models of myocardial infarction. Ascribing any therapeutic benefit to an effect on neovascularization may require conditionally knocking down (or out) specific cytokines in the transplanted cell population, where one would lose the presumed therapeutic effect on cardiac function as well as on neoangiogenesis. Second, if it is indeed a paracrine effect, an examination of the relative efficacy of the intramyocardial delivery of angiogenic cytokines in experimental model systems of acute myocardial infarction appears warranted. If indeed the effects of cell therapy in the acutely ischemic heart are not related to transdifferentiation, it could become valuable to define which autologous cell type has the optimal profile of angiogenic paracrine factors to drive the desired neovascularization end point. It will also become of interest to determine if there is a distinct functional difference and role for the previously resident c-kit ${ }^{+}$cells in the heart (17) versus the influx of c-kit ${ }^{+}$cells from the bone marrow. In this regard, further characterization, purification, and functional analyses of specific cell subsets could be informative.

On the clinical front, these studies raise the larger question as to whether the mechanistic basis for cardiovascular cell therapy following acute myocardial infarction might be more closely related to the biology of wound healing than regenerative medicine. The short-term effects of infiltrating or transplanted cell types from bone marrow might be partially based on the triggering of an inflammatory response, with the secondary release of cytokines and nonspecific angiogenic cues. In a similar manner, homing of these cells into the injured myocardium might represent the migration of inflammatory cells across a breakdown in the vascular permeability barrier that accompanies many forms of tissue injury. Interestingly, one of the clear messages from the placebo-controlled trials is that there can be a statistically significant, partial recovery of cardiac function in control groups (5). In the only long-term study to date, the control group eventually reached the same extent of functional recovery seen in the treated groups (2), which might relate to the acceleration of cardiac scar formation by inflammatory pathways. Inflammation is known to be linked to atherogenesis, and recent studies suggest luminal loss in the infarct-related artery following intracoronary delivery of bone marrow precursors (18). Accordingly, careful clinical monitoring for these long-term effects seems warranted. 


\section{A parable for regenerative medicine}

In this ongoing parable of regenerative medicine, the initial hope was that we were in an advantageous position to take a "shot on goal" by moving quickly to clinical studies. The rationale was noble, clear, and compelling, particularly given the unmet clinical need and the robust results of early scientific studies. However, it now is becoming increasingly clear that we may not have the optimal cell type in hand, let alone a clear understanding of other key variables such as in vivo delivery, efficiency of grafting, and suppression of alternative, unwanted cell phenotypes (e.g., pacemaker cell formation in the midst of cardiac muscle regeneration). Cardiovascular stem cell biology still remains one of the most intriguing fields of scientific inquiry in the cardiovascular field and holds great long-term potential. Perhaps, given our growing understanding of the complexity of cell therapy for heart disease, the time has come to "move the ball down the field" by first trying to understand the mechanistic basis of this potential therapy.

Address correspondence to: Kenneth R. Chien, MGH Cardiovascular Research Center, Massachusetts General Hospital,
Richard B. Simches Research Center/CPZN 3208, 185 Cambridge Street, Boston, Massachusetts 02114-2790, USA. Phone: (617) 643-3440; Fax: (617) 643-3451: E-mail: kchien@partners.org.

1. Assmus, B., et al. 2002. Transplantation of Progenitor Cells and Regeneration Enhancement in Acute Myocardial Infarction (TOPCARE-AMI). Circulation. 106:3009-3017.

2. Meyer, G.P., et al. 2006. Intracoronary bone marrow cell transfer after myocardial infarction: eighteen months' follow-up data from the randomized, controlled BOOST (BOne marrOw transfer to enhance ST-elevation infarct regeneration) trial. Circulation. 113:1287-1294.

3. Janssens, S., et al. 2006. Autologous bone marrow-derived stem-cell transfer in patients with ST-segment elevation myocardial infarction: double-blind, randomised controlled trial. Lancet. 367:113-121.

4. Lunde, K., et al. 2005. Autologous stem cell transplantation in acute myocardial infarction: the ASTAMI randomized controlled trial. Intracoronary transplantation of autologous mononuclear bone marrow cells, study design and safety aspects. Scand. Cardiovasc. J. 39:150-158.

5. Zohlnhofer, D., et al. 2006. Stem cell mobilization by granulocyte colony-stimulating factor in patients with acute myocardial infarction: a randomized controlled trial. JAMA. 295:1003-1010.

6. Cleland, J.G., Freemantle, N., Coletta, A.P., and Clark, A.L. 2006. Clinical trials update from the American Heart Association: REPAIR-AMI, ASTAMI, JELIS, MEGA, REVIVE-II, SURVIVE, and PROACTIVE. Eur. J. Heart Fail. 8:105-110.
7. Wollert, K.C., and Drexler, H. 2006. Cell-based therapy for heart failure. Curr. Opin. Cardiol. 21:234-239.

8. Orlic, D., et al. 2001. Mobilized bone marrow cells repair the infarcted heart, improving function and survival. Proc. Natl. Acad. Sci. U. S. A. 98:10344-10349.

9. Orlic, D., et al. 2001. Bone marrow cells regenerate infarcted myocardium. Nature. 410:701-705.

10. Hofmann, M., et al. 2005. Monitoring of bone marrow cell homing into the infarcted human myocardium. Circulation. 111:2198-2202.

11. Murry, C.E., et al. 2004. Haematopoietic stem cells do not transdifferentiate into cardiac myocytes in myocardial infarcts. Nature. 428:664-668.

12. Balsam, L.B., et al. 2004. Haematopoietic stem cells adopt mature haematopoietic fates in ischaemic myocardium. Nature. 428:668-673.

13. Nygren, J.M., et al. 2004. Bone marrow-derived hematopoietic cells generate cardiomyocytes at a low frequency through cell fusion, but not transdifferentiation. Nat. Med. 10:494-501.

14. Dimmeler, S., Zeiher, A.M., and Schneider, M.D. 2005. Unchain my heart: the scientific foundations of cardiac repair. J. Clin. Invest. 115:572-583. doi:10.1172/JCI200524283

15. Chien, K.R. 2004. Stem cells: lost in translation. Nature. 428:607-608.

16. Fazel, S., et al. 2006. Cardioprotective c-kit ${ }^{+}$cells are from the bone marrow and regulate the myocardial balance of angiogenic cytokines. J. Clin. Invest. 116:1865-1877. doi:10.1172/JCI27019.

17. Beltrami, A.P., et al. 2003. Adult cardiac stem cells are multipotent and support myocardial regeneration. Cell. 114:763-776.

18. Mansour, S., et al. 2006. Intracoronary delivery of hematopoietic bone marrow stem cells and luminal loss of the infarct-related artery in patients with recent myocardial infarction. J. Am. Coll. Cardiol. 47:1727-1730.

\title{
The leak stops here: platelets as delivery vehicles for coagulation factors
}

\author{
Katherine A. High \\ Howard Hughes Medical Institute, The Children's Hospital of Philadelphia, Philadelphia, Pennsylvania, USA.
}

\begin{abstract}
Gene therapy is an attractive approach for the treatment of hemophilia, as continuous expression of donated clotting factor VIII (FVIII) DNA would ensure clotting factor replacement at constant circulating levels rather than at the peaks and troughs that characterize the current protein infusion therapeutic approach. In this issue of the JCI, Shi et al. describe an interesting variant of a gene transfer approach for hemophilia (see the related article beginning on page 1974). They show that targeted expression of FVIII in megakaryocytes, with storage in the $\alpha$-granules of platelets, has the advantage of delivering clotting factors directly to the site of an injury, where platelets accumulate in large numbers and undergo activation accompanied by release of granule contents. Earlier clinical experience with gene transfer into hematopoietic cells highlighted the potential safety risks of this approach, but an $F 8$ transgene may represent a lower risk than transgenes for growth factors or their receptors.
\end{abstract}

Nonstandard abbreviations used: FVIII, factor VIII. Conflict of interest: The author holds issued patents related to the use of adeno-associated virus for the treatment of hemophilia.

Citation for this article: J. Clin. Invest. 116:1840-1842 (2006). doi:10.1172/JCI29193.
Gene transfer for the treatment of genetic disease remains one of the most compelling ideas in modern molecular medicine. In genetic disease, where the therapeutic objective is long-term expression of a specific protein, there are - broadly speaking - two potential strategies: gene transfer into a stem cell via an integrating vector, so that all daughter cells contain the genetic modification, and gene transfer with a nonintegrating vector into long-lived postmitotic cells (such as skeletal or cardiac muscle, hepatocytes, or cells in the central nervous system), so that long-lasting expression is achieved even without vector integration. Both of these strategies have been used to achieve long-term expression of a clotting factor, with phenotypic correction, in large animal models of hemophilia, one by use of a retroviral vector in neonatal (rapidly dividing) hepatocytes (1), and the other by use of an adeno-associated virus vector introduced into mature hepatocytes (2-4) (Figure 1A).

One of the advantages of hemophilia as a model for gene therapy is that tissue-specific expression of the donated gene is not 